\title{
Instrumentos para evaluar la calidad percibida por los usuarios en los servicios de salud*
}

\author{
Instruments to Evaluate Quality Perceived by Health Service Users \\ Instrumentos para avaliar a qualidade percebida pelos usuários dos serviços de saúde
}

\author{
Daniel Eduardo Henao Nieto ${ }^{\text {a }}$ \\ Universidad de Antioquia, Colombia \\ ORCID: http://orcid.org/0000-0002-7419-6080 \\ Adriana Giraldo Villa \\ Clínica del Hospital Pablo Tobón Uribe, Colombia \\ ORCID: http://orcid.org/0000-0001-6204-6897 \\ Carlos Enrique Yepes Delgado \\ Universidad de Antioquia, Colombia \\ Clínica del Hospital Pablo Tobón Uribe, Colombia \\ ORCID: http://orcid.org/0000-0001-5656-4989
}

DOI: https://doi.org/10.11144/Javeriana.rgps17-34.iecp

\section{Resumen:}

La calidad es considerada un factor clave en la diferenciación y en la excelencia de los servicios de salud, y la percepción de un paciente sobre la prestación del servicio determina en forma definitiva su nivel de calidad. Se han descrito indicadores que ayudan a evaluar la calidad de los servicios de salud, entre estos, indicadores de efectividad, de oportunidad y de adhesión. Sin embargo, la evaluación integral de la calidad percibida en los servicios de salud es aún compleja. Se realizó una búsqueda en Medline, Embase, OVID, Lilacs, Ebsco, Scielo y The Cochrane Library para identificar instrumentos de tipo cualitativo o cuantitativo que permitieran medir la calidad de los servicios de salud percibida por los pacientes. Se encontraron estudios que validaron y aplicaron la escala Servqual y Servqhos en el ámbito hospitalario. Adicionalmente, en nuestro país se intentó validar escalas diferentes a estas, pero no contaron con un rigor estadístico importante.

Palabras clave: calidad de la atención de salud, indicadores de calidad de la atención de salud, evaluación de la calidad de atención de salud, evaluación de servicios de salud, evaluación de los servicios, estudios de validación.

\section{Abstract:}

Quality is considered as a key factor for the differentiation and excellence of the health services. The way patients perceive the health care service determines conclusively the service quality levels. This work describes some indicators that help to assess the health service quality including effectiveness, opportunity, and adherence indicators. However, the comprehensive evaluation of the quality as perceived in the health services is still a complex matter. A search was conducted in the databases Medline, Embase, OVID, Lilacs, Ebsco, Scielo and The Cochrane Library in order to identify those instruments, either qualitative or quantitative, allowing to measure the health service quality as perceived by the patients. Studies were found that validated and applied the scales Servqual and Servqhos in the hospital environment. Finally, there were some attempts to validate some scales different to these ones, but they were not applied with a significant statistic rigorousness.

Keywords: health care quality, quality indicators in health care, quality evaluation of health care, health service evaluation, service evaluation, validation studies.

\section{Resumo:}

A qualidade é considerada fator chave na diferenciação e excelência dos serviços de saúde e a percepção de um paciente sobre a prestação do serviço determina em forma definitiva seu nível de qualidade. Indicadores que ajudam a avaliar a qualidade dos serviços de saúde já foram descritos, entre estes, indicadores de efetividade, de oportunidade e de adesão. No entanto, a avaliação integral da qualidade percebida nos serviços de saúde é ainda complexa. Realizou-se uma busca em Medline, Embase, OVID, Lilacs, Ebsco, Scielo e The Cochrane Library para identificar instrumentos de tipo qualitativo ou quantitativo que permitiram medir a qualidade dos serviços de saúde percebida pelos pacientes. Encontraram-se estudos que validaram e aplicaram a escala Servqual e Servqhos

a Autor de correspondencia. Correo electrónico: dhenaonieto@gmail.com 
no âmbito hospitalar. Além disso, em nosso país tentou-se validar escalas diferentes a estas, mas não contamos com rigor estatístico importante.

Palavras-chave: qualidade da atenção de saúde, indicadores de qualidade da atenção de saúde, avaliação da qualidade de atenção de saúde, avaliação de serviços de saúde, avaliação dos serviços, estudos de validação.

\section{Introducción}

La calidad en los servicios de salud se ha convertido en un tema relevante desde la década de los ochenta, esto dado por el aumento de los usuarios y su participación en los servicios de salud. Estos servicios deben orientarse a las necesidades de los pacientes y de esta forma ofrecer nuevos enfoques y soluciones a las demandas de los usuarios. La calidad además es considerada un factor clave en la diferenciación y excelencia de los servicios, ya que los centros de salud pueden prestar servicios similares, pero con diferente calidad, lo que genera una ventaja distintiva en los usuarios. La mejora en la calidad de los servicios de salud influye de forma decisiva en el número de pacientes satisfechos y por la tanto en la lealtad del paciente hacia el centro de salud $[1,2]$.

Algunos autores definen la calidad de los servicios de salud como los servicios prestados al usuario de una forma accesible y equitativa, en las mejores condiciones científicas, profesionales e institucionales para satisfacer y adherir al usuario [3]; este es un concepto que se ha ido construyendo a lo largo de los años. La conceptualización de la calidad en salud inicia con una perspectiva técnica considerada como la habilidad de alcanzar objetivos deseables, haciendo uso de medios legítimos. Esta definición relega a un plano inferior la perspectiva de los pacientes en cuanto al servicio que se les presta. Más tarde se reconoce que la percepción de un paciente sobre la prestación del servicio determina, en forma definitiva, su nivel de calidad. Esta definición vincula una visión personal y subjetiva de la calidad y reconoce que el usuario a partir de interacciones humanas experimenta sensaciones agradables y desagradables que se traducen en sentimientos de satisfacción o insatisfacción [4].

Este último enfoque implica incluir el juicio subjetivo del usuario dentro de la evaluación, de ahí que se empieza a reconocer el concepto de calidad del servicio percibido, en el cual la evaluación está basada fundamentalmente en las discrepancias entre las expectativas generadas en los usuarios y sus percepciones respecto al servicio recibido $[5,6,7]$.

Pese a la dificultad en medir la percepción de los pacientes, se han identificado ciertos indicadores que ayudan a evaluar los servicios de salud, entre estos tenemos: indicadores de efectividad, que se expresan en la percepción de los pacientes en satisfacer necesidades de atención con servicios generales, electivos especializados o de urgencias, la bondad clínica de las intervenciones, cumplir las expectativas generadas y mantener o recuperar la salud. De oportunidad, que se expresa en la percepción de tiempo de espera desde que llega a la institución, desde que se llega a urgencias, o desde que se aprueba su atención sin cita previa. De adhesión, que se manifiesta como la intención favorable o no del paciente para regresar a la institución solicitando el mismo servicio u otro diferente. Otros factores incluyen el trato recibido por el personal administrativo o asistencial, las características estético-funcionales, además del aseo de la institución, la comunicación con el usuario, la atención durante hospitalización, la confianza percibida por el paciente para expresar sus requerimientos e inquietudes, entre otros [3].

A pesar de los indicadores anteriormente descritos, aún es compleja una evaluación integral de la calidad percibida en los servicios de salud. Por ello, el objetivo de esta revisión fue describir el estado actual de los instrumentos desarrollados para evaluar la calidad de la atención en salud desde la perspectiva de los usuarios, así como explorar su capacidad para ser adaptados a nuestro entorno. 


\section{Método}

Se realizó una revisión descriptiva de los artículos, guías de práctica clínica, tesis de posgrado y demás documentos donde se referenciaran instrumentos cualitativos o cuantitativos para la medición de la calidad de los servicios de salud desde la perspectiva del usuario.

Los artículos se seleccionaron teniendo en cuenta su pertinencia con el objetivo anteriormente descrito, de acuerdo con el título, los autores, los resúmenes y los resultados de los artículos que se encontraron en el proceso de búsqueda. Se incluyeron artículos que fueron publicados desde 1980, ya que el fuerte de publicaciones sobre el tema de calidad en servicios de salud se presentó a finales de los años ochenta y principios de los noventa; no se hicieron discriminaciones por el tipo de artículos, se dio mayor prevalencia a aquellos estudios donde se validaban las escalas en población colombiana, aunque esto no fue un criterio de exclusión para el análisis de otros artículos. En algunas bases de datos que lo permitían, la estrategia de búsqueda se realizó en español, esto con el fin de poder seleccionar artículos de la región que hablaran sobre el tema.

La búsqueda se realizó en las siguientes bases de datos electrónicas: Medline, Embase, OVID, Lilacs, Ebsco, y Scielo, The Cochrane Library hasta la fecha de junio de 2016, con la pregunta: ¿Existen escalas validadas cualitativas y cuantitativas para evaluar la calidad percibida en los servicios de salud por parte de los pacientes? Las palabras clave usadas para la búsqueda fueron: quality, healthcare, patients, hospital, perception, validation studies, studies quantitatives, studies qualitatives, scale, quality assurance, evaluation services.

Para la estrategia de búsqueda (tabla 1), dada la similitud conceptual entre los constructos de calidad de servicio y satisfacción, fue usual encontrar durante la revisión instrumentos para medir satisfacción o experiencia de la atención recibida. Estos instrumentos fueron excluidos del análisis, ya que la literatura expone que los dos conceptos no son equivalentes y que la satisfacción se puede considerar una evaluación susceptible de ser cambiada con cada experiencia de consumo, mientras que la calidad percibida supone una evaluación más global y estable en el tiempo $[8,9,10,11]$.

Además, se indagó por artículos, documentos o escalas en las páginas web de asociaciones reconocidas a nivel internacional que se encargan de evaluar y certificar en lo relacionado con la calidad del servicio de salud. Se hizo énfasis en The Agency for Health Care Research and Quality (AHRQ), organización que suministró el software por medio del cual se evalúan los mejores hospitales en Estados Unidos. 
TABLA 1

Estrategia de búsqueda en las diferentes bases de datos, hallazgos más representativos

\begin{tabular}{|l|c|}
\hline \multicolumn{2}{|c|}{ Fecha de búsqueda: Junio de 2016} \\
\hline Pregunta & $\begin{array}{c}\text { ¿Existen escalas validadas cualitativas y cuantitativas para evaluar la calidad percibida en bs servicios de salud por parte } \\
\text { de los pacientes? }\end{array}$ \\
$\begin{array}{l}\text { Palabras } \\
\text { clave }\end{array}$ & $\begin{array}{c}\text { quality, healthcare, patients, hospital, perception, validation studies, studies quantitatives, studies qualitatives, scale, } \\
\text { quality assurance, evaluation services. }\end{array}$ \\
\hline
\end{tabular}

\begin{tabular}{|c|c|c|c|c|}
\hline \multicolumn{5}{|c|}{ Bases de datos electrónicas consultadas y hallazgos } \\
\hline \multicolumn{5}{|c|}{ MEDLINE, plataforma pubmed } \\
\hline Search & $\begin{array}{l}\text { Add to } \\
\text { builder }\end{array}$ & Query & $\begin{array}{l}\text { Items } \\
\text { found }\end{array}$ & Time \\
\hline$\# 21$ & Add & $\begin{array}{l}\text { Search (Patient Satisfaction) AND health care quality assessment [MeSH } \\
\text { Terms] Filters: Publication date from } 2005 / 01 / 01 \text { to } 2103 / 12 / 31\end{array}$ & $\underline{2990}$ & $10: 30: 11$ \\
\hline$\# 20$ & Add & $\begin{array}{l}\text { Search ((evaluation) AND service quality) AND hospital Filters: Publication date } \\
\text { from } 2005 / 01 / 01 \text { to } 2103 / 12 / 31\end{array}$ & 3626 & $10: 29: 07$ \\
\hline$\# 18$ & Add & $\begin{array}{l}\text { Search Health Service Quality Scale: Brazilian Portuguese translation, reliability and } \\
\text { validity Filters:Publication date from } 2005 / 01 / 01 \text { to } 2103 / 12 / 31\end{array}$ & 4 & $10: 28: 17$ \\
\hline$\# 17$ & Add & $\begin{array}{l}\text { Search (((perception) AND quality) AND healthcare) AND } \\
\text { hospital Filters: Publication date from } 2005 / 01 / 01 \text { to } 2103 / 12 / 31\end{array}$ & 1590 & $10: 27: 25$ \\
\hline$\# 16$ & Add & $\begin{array}{l}\text { Search ((validation studies[MeSH Terms]) AND health care quality[MeSH } \\
\text { Terms]) AND hospital[MeSH Terms] Filters: Publication date from 2005/01/01 to } \\
2103 / 12 / 31\end{array}$ & $\underline{0}$ & $10: 25: 37$ \\
\hline$\# 15$ & Add & $\begin{array}{l}\text { Search ((health services evaluation [MeSH Terms]) AND health care quality } \\
\text { [MeSH Terms] AND ( "2005/01/01"[PDat] : "2103/12/31"[PDat] } \\
\text { ))) Filters: Publication date from } 2005 / 01 / 01 \text { to } 2103 / 12 / 31\end{array}$ & 60705 & $10: 23: 37$ \\
\hline \#14 & Add & $\begin{array}{l}\text { Search ((validation studies[MeSH Terms]) AND health care quality[MeSH } \\
\text { Terms]) AND rating scale Schema Filters: Publication date from 2005/01/01 to } \\
2103 / 12 / 31\end{array}$ & $\underline{0}$ & 10:19:08 \\
\hline$\# 11$ & Add & $\begin{array}{l}\text { Search health care quality indicators[MeSH Terms] Filters: Publication date from } \\
2005 / 01 / 01 \text { to } 2103 / 12 / 31\end{array}$ & 25123 & $10: 10: 12$ \\
\hline$\# 10$ & Add & $\begin{array}{l}\text { Search ((patients) AND perceived) AND health care quality[MeSH } \\
\text { Terms] Filters: Publication date from } 2005 / 01 / 01 \text { to } 2103 / 12 / 31\end{array}$ & $\underline{50083}$ & $10: 08: 57$ \\
\hline$\# 9$ & Add & $\begin{array}{l}\text { Search (health services evaluation [MeSH Terms] AND health care quality [MeSH } \\
\text { Terms]) Filters:Publication date from } 2005 / 01 / 01 \text { to } 2103 / 12 / 31\end{array}$ & $\underline{60705}$ & $10: 05: 25$ \\
\hline \#6 & Add & $\begin{array}{l}\text { Search (patients [MeSH Terms] AND health care quality [ MeSH } \\
\text { Terms]) Filters: Publication date from } 2005 / 01 / 01 \text { to } 2103 / 12 / 31\end{array}$ & 17269 & $10: 02: 22$ \\
\hline$\# 3$ & Add & $\begin{array}{l}\text { Search "Quality of HeathCare/statistics and numerical data" } \\
\text { [Majr] Filters: Publication date from } 2005 / 01 / 01 \text { to } 2103 / 12 / 31\end{array}$ & 22485 & $9: 55: 31$ \\
\hline$\# 2$ & Add & Search quality healthcare Filters: Publication date from $2005 / 01 / 01$ to $2103 / 12 / 31$ & 29722115 & $9: 50: 03$ \\
\hline$\# 1$ & Add & Search quality healthcare & 5597677 & $9: 48: 52$ \\
\hline
\end{tabular}


TABLA 1 (CONT.)

Estrategia de búsqueda en las diferentes bases de datos, hallazgos más representativos

\begin{tabular}{|c|c|c|}
\hline \multicolumn{3}{|c|}{ EMBASE - Excerpta Medica dataBase } \\
\hline No. & Query & Resulks \\
\hline \#10 & \#4 AND \#9 & 1 \\
\hline$\$ 9$ & servqual AND [2005-2016]/py & 110 \\
\hline$\# 8$ & $\begin{array}{l}\text { perceived AND quality AND healtheareand AND 'patient'/exp AND 'hospital'/exp AND scales } \\
\text { AND [2005-2016]/py }\end{array}$ & 0 \\
\hline$\$ 7$ & $\begin{array}{l}\text { perceived AND quality AND healtheareand AND 'patients'/exp AND 'hospital'/exp AND rating } \\
\text { AND scales AND [2005-2016/py }\end{array}$ & 0 \\
\hline$\$ 6$ & $\begin{array}{l}\text { perceived AND quality AND 'healtheare /exp AND 'patients'/exp AND 'hospital/exp AND } \\
\text { validation AND studies AND }[2005-2016 \mathrm{\gamma} \text { py }\end{array}$ & 4 \\
\hline$\# 5$ & $\begin{array}{l}\text { 'measurement/exp AND quality AND 'healthcare'/exp AND perceived AND 'patients'/exp } \\
\text { AND [2005-2016]/py }\end{array}$ & 97 \\
\hline$\# 4$ & $\begin{array}{l}\text { perceived AND quality AND 'healtheare'/exp AND 'patients'/exp AND 'hospital/exp AND } \\
{[2005-2016] \text { py }}\end{array}$ & 1164 \\
\hline$\# 3$ & $\begin{array}{l}\text { quality AND 'healthcare'/exp AND 'perception'/exp AND 'patients'/exp AND validation AND } \\
\text { studies AND [2005-2016]/py }\end{array}$ & 4 \\
\hline$\# 2$ & $\begin{array}{l}\text { quality AND 'health'/exp AND care AND 'perception'/exp AND 'patients'/exp AND [2005- } \\
\text { 2016]/py }\end{array}$ & 105 \\
\hline$\# 1$ & quality AND of AND ('bealth'/exp OR health) AND care AND [2005-2016]/py & 283505 \\
\hline
\end{tabular}


TABLA 1 (CONT.)

Estrategia de búsqueda en las diferentes bases de datos, hallazgos más representativos

\begin{tabular}{|c|c|c|c|}
\hline \multicolumn{4}{|c|}{ OVID } \\
\hline$\# \mathbf{\nabla}$ & Searches & Results & SearchType \\
\hline 16 & (perceived and quality and healthcare and quantitative studies).mp. [mp=tx, bt, ti, ab, ct] & 1117 & Advanced \\
\hline 15 & limit 14 to $y r=" 2008-2016^{\prime \prime}$ & 185 & Advanced \\
\hline 14 & (scak rating and quality and healthcare).mp. [mp=tx, bt, ti, ab, ct] & 312 & Advanced \\
\hline 13 & limit 12 to $\mathrm{yr}^{-1}=2008-2016^{\prime \prime}$ & 26842 & Advanced \\
\hline 12 & (patients and satisfaction and quality and healthcare).mp. [mp=tx, bt, ti, ab, ct] & 41078 & Advanced \\
\hline 11 & Imit 10 to $y=" 1998-2016^{\prime \prime}$ & 11239 & Advanced \\
\hline 10 & (measurement and quality and healthcare and perceived and patients).mp. [mp=tx, bt, ti, ab, ct] & 11384 & Advanced \\
\hline 9 & (perception and quality and hethcare and patients and hospital).mp. [mp=tx, bt, ti, ab, ct] & 0 & Advanced \\
\hline 8 & limit 7 to $y \mathrm{r}=" 2008-2016^{\prime \prime}$ & 5563 & Advanced \\
\hline 7 & healthcare AND qualty AND servicies evaluation \{Including Limited Related Terms\} & 7332 & Basic \\
\hline 6 & percieved AND quality AND bealtheare AND patients \{Including Limited Rekated Terms\} & 7547 & Basic \\
\hline 5 & limit 4 to $\mathrm{yr}^{=1} 1998-2016^{\circ}$ & 6093 & Advanced \\
\hline 4 & qualify AND healthcare AND validation studies \{Including Limited Related Terms\} & 6212 & Basic \\
\hline 2 & Imit 1 to $\mathrm{yr}=" 1998-2016 "$ & 6583 & Advanced \\
\hline 1 & qualify AND healtheare \{Including Limited Rekated Terms\} & 6785 & Basic \\
\hline
\end{tabular}

TABLA 1 (CONT.)

Estrategia de búsqueda en las diferentes bases de datos, hallazgos más representativos

\begin{tabular}{|c|c|}
\hline \multicolumn{2}{|l|}{ LILACS } \\
\hline Estrategia de Búsqueda & Resultados \\
\hline tw(calidad percibida en salud) AND dbx("LILACS") & 95 \\
\hline (twr(calidad percibida en salud) AND mj("Calidad de la Atención de Salud") AND type("article")) & 12 \\
\hline $\begin{array}{l}\text { (tw:(calidad percibida en salud) AND dbr("LILACS")) AND mj:("Calidad de la Atención de Salud" OR "Satisfacción del } \\
\text { Paciente") AND type:("article") }\end{array}$ & 13 \\
\hline $\begin{array}{l}\text { (tw:(calidad percibida en salud )) AND (tw:(escahs )) AND mj:("Calidad de la Atención de Salud" OR "Satisfacción del } \\
\text { Paciente") }\end{array}$ & 1 \\
\hline
\end{tabular}


TABLA 1 (CONT.)

Estrategia de búsqueda en las diferentes bases de datos, hallazgos más representativos

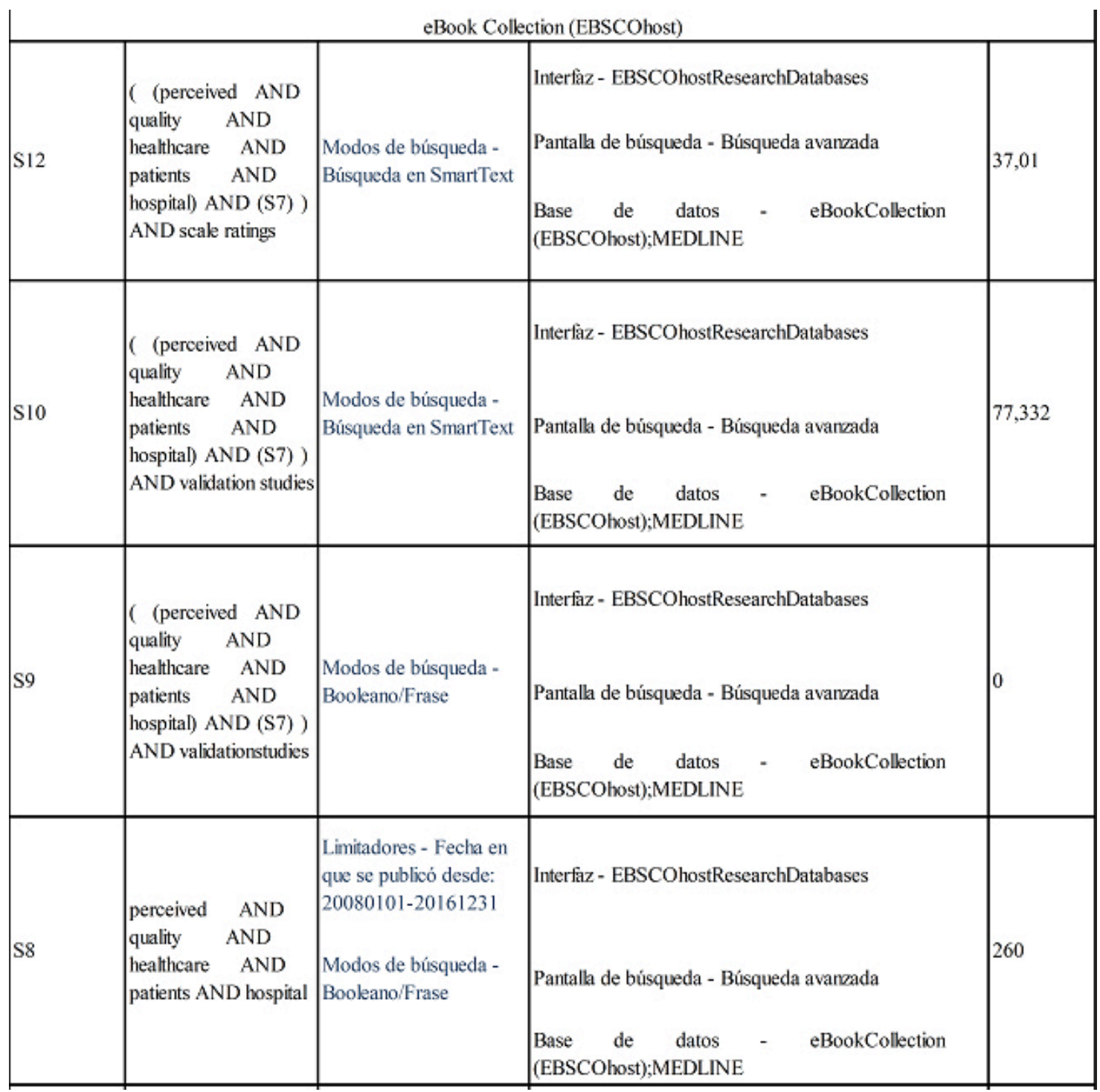


TABLA 1 (CONT.)

Estrategia de búsqueda en las diferentes bases de datos, hallazgos más representativos

eBook Collection (EBSCOhost)

\begin{tabular}{|c|c|c|c|c|}
\hline S7 & $\begin{array}{lc}\text { perceived } & \text { AND } \\
\text { quality } & \text { AND } \\
\text { healthcare } & \text { AND } \\
\text { patients } & \end{array}$ & $\begin{array}{l}\text { Limitadores - Fecha en } \\
\text { que se publicó desde: } \\
\text { 20080101-20161231 } \\
\text { Modos de búsqueda - } \\
\text { Booleano/Frase }\end{array}$ & $\begin{array}{l}\text { Interfaz - EBSCOhostResearchDatabases } \\
\text { Pantalla de búsqueda - Búsqueda avanzada } \\
\text { Base de datos - eBookCollection } \\
\text { ERSCOhost):MFDLINF }\end{array}$ & 719 \\
\hline S6 & $\begin{array}{ll}\text { qualitative } & \text { AND } \\
\text { perception } & \text { AND } \\
\text { quality } & \text { AND } \\
\text { healthcare } & \end{array}$ & $\begin{array}{l}\text { Limitadores - Fecha en } \\
\text { que se publicó desde: } \\
20080101-20161231 \\
\text { Modos de búsqueda - } \\
\text { Booleano/Frase }\end{array}$ & $\begin{array}{l}\text { Interfaz - EBSCOhostResearchDatabases } \\
\text { Pantalla de búsqueda - Búsqueda avanzada } \\
\text { Base de datos - eBookCollection } \\
\text { (EBSCOhost);MEDLINE }\end{array}$ & 143 \\
\hline S5 & $\begin{array}{l}\text { quantitative AND } \\
\text { perception AND } \\
\text { quality AND } \\
\text { healthcare }\end{array}$ & $\begin{array}{l}\text { Limitadores - Fecha en } \\
\text { que se publicó desde: } \\
20080101-20161231 \\
\text { Modos de búsqueda - } \\
\text { Booleano/Frase }\end{array}$ & $\begin{array}{l}\text { Interfaz - EBSCOhostResearchDatabases } \\
\text { Pantalla de búsqueda - Búsqueda avanzada } \\
\text { Base de datos - eBookCollection } \\
\text { (EBSCOhost);MEDLINE }\end{array}$ & 46 \\
\hline S4 & $\begin{array}{l}\text { perceivedquality AND } \\
\text { healthcare }\end{array}$ & $\begin{array}{l}\text { Limitadores - Fecha en } \\
\text { que se publicó desde: } \\
20080101-20161231 \\
\text { Modos de búsqueda - } \\
\text { Booleano/Frase }\end{array}$ & $\begin{array}{l}\text { Interfaz - EBSCOhostResearchDatabases } \\
\text { Pantalla de búsqueda - Búsqueda avanzada } \\
\text { Base de datos - eBookCollection } \\
\text { (EBSCOhost);MEDLINE }\end{array}$ & 333 \\
\hline
\end{tabular}


TABLA 1 (CONT.)

Estrategia de búsqueda en las diferentes bases de datos, hallazgos más representativos

\begin{tabular}{|c|c|c|c|c|c|}
\hline S3 & $\begin{array}{l}\text { quality } \\
\text { healthcare }\end{array}$ & AND & $\begin{array}{l}\text { Limitadores - Fecha en } \\
\text { que se publicó desde: } \\
20080101-20161231 \\
\text { Modos de búsqueda - } \\
\text { Booleano/Frase }\end{array}$ & $\begin{array}{l}\text { Interlaz - EBSCOhostResearchDatabases } \\
\text { Pantalla de búsqueda - Búsqueda avanzada } \\
\text { Base de datos - eBookCollection } \\
\text { (EBSCOhost);MEDLINE }\end{array}$ & 30,199 \\
\hline S2 & $\begin{array}{l}\text { quality } \\
\text { healthcare }\end{array}$ & AND & $\begin{array}{l}\text { Modos de búsqueda - } \\
\text { Bookeano/Frase }\end{array}$ & $\begin{array}{l}\text { Interfaz - EBSCOhostResearchDatabases } \\
\text { Pantalla de búsqueda - Búsqueda avanzada } \\
\text { Base de datos - eBookCollection } \\
\text { (EBSCOhost);MEDLINE }\end{array}$ & 44,478 \\
\hline S1 & $\begin{array}{l}\text { perception } \\
\text { quality } \\
\text { healthcare }\end{array}$ & $\begin{array}{l}\text { AND } \\
\text { AND }\end{array}$ & $\begin{array}{l}\text { Modos de búsqueda - } \\
\text { Booleano/Frase }\end{array}$ & $\begin{array}{l}\text { Interfáz - EBSCOhostResearchDatabases } \\
\text { Pantalk de búsqueda - Búsqueda avanzada } \\
\text { Base de datos - eBookCollection } \\
\text { (EBSCOhost);MEDLINE }\end{array}$ & 933 \\
\hline
\end{tabular}


TABLA 1 (CONT.)

Estrategia de búsqueda en las diferentes bases de datos, hallazgos más representativos

\begin{tabular}{|c|c|c|c|}
\hline \multicolumn{4}{|c|}{ SCIELO } \\
\hline$\#$ & \multicolumn{2}{|l|}{ Búsqueda } & Resultados \\
\hline 10 & \multicolumn{2}{|l|}{ (perceived) AND (quality) AND (health care) AND (quantitative studies) } & 3 \\
\hline 9 & \multicolumn{2}{|l|}{ (perceived) AND (quality) AND (health care) AND (qualitative studies) } & 10 \\
\hline 8 & \multicolumn{2}{|l|}{ (perceived) AND (quality) AND (health care) AND (validation studies) } & 0 \\
\hline 7 & \multicolumn{2}{|l|}{ (perceived) AND (quality) AND (health care) AND (rating scales) } & 0 \\
\hline 6 & \multicolumn{2}{|l|}{ (perceived) AND (quality) AND (healthcare) } & 50 \\
\hline 5 & \multicolumn{2}{|l|}{ (quality) AND (healthcare) } & 987 \\
\hline 4 & \multicolumn{2}{|l|}{ (satisfacción) AND (pacientes) AND (escalas) } & 25 \\
\hline 3 & \multicolumn{2}{|l|}{ (calidad percibida en salud) AND (hospitales) } & 9 \\
\hline 2 & \multicolumn{2}{|l|}{ (percepción AND calidad AND atención en salud AND escalas) } & 6 \\
\hline 1 & \multicolumn{2}{|l|}{ (calidad percibida en salud) } & 126 \\
\hline \multicolumn{4}{|c|}{ COCHRANE LIBRARY } \\
\hline$\# \mathbf{\nabla}$ & Searches & Results & SearchType \\
\hline 5 & "MeSH descriptor: [PatientAcceptance of HealthCare] explodealltrees in Cochrane Reviews" & 160 & Advanced \\
\hline 4 & $\begin{array}{l}\text { measurement and quality and healthcare and perceived and patients in Title, Abstract, } \\
\text { Keywords in Cochrane Reviews' }\end{array}$ & 2 & Advanced \\
\hline 3 & $\begin{array}{l}\text { perceived AND quality AND healthcare AND quantitative studies in Tille, Abstract, } \\
\text { Keywords in Trials' }\end{array}$ & 3 & Advanced \\
\hline 2 & $\begin{array}{l}\text { "MeSH descriptor: [HealthCareQuality, Access, and Evaluation] explode all trees in Cochrane } \\
\text { Reviews }\end{array}$ & 5571 & Advanced \\
\hline 1 & Quality of Health Care in Title, Abstract, Keywords in Cochrane Reviews ' & 430 & Advanced \\
\hline
\end{tabular}

\section{Resultados}

Con respecto a la calidad de servicio percibida, hubo una escala que siempre fue constante en la mayoría de los artículos, la escala Servqual. El modelo Servqual fue creado por Zeithaml, Parasuraman y Berry a finales de la década de los ochenta, para evaluar las percepciones de los clientes sobre la calidad del servicio en las organizaciones comerciales, pero con el tiempo ha sido validada en diferentes países para evaluar la calidad en los servicios de salud. La escala Servqual tiene cinco dimensiones de calidad, que son compartidas por las diferentes organizaciones que prestan servicios en general, estas dimensiones según sus autores son: fiabilidad, capacidad de respuesta, seguridad, empatía y elementos tangibles. La evaluación de estas dimensiones está distribuida en veintidós preguntas de percepciones y expectativas de los usuarios. El tipo de respuesta sé da en una escala tipo Likert [11].

A partir de este estudio, a través de los años se han realizado en diferentes países escalas modificadas para evaluar calidad en servicios de salud basadas en el esquema general de Servqual. A continuación, se presenta el resumen de los resultados de algunos artículos relevantes sobre validación de instrumentos para evaluar la 
calidad percibida por los usuarios en los servicios de salud. Se presentarán bajo la lógica de autor, año, lugar/ entorno, objetivo, instrumento, metodología, idioma y resultados (tabla 2).

TABLA 2

Resumen de los resultados de algunos artículos relevantes sobre validación de instrumentos para evaluar la calidad percibida por los usuarios en los servicios de salud.

\begin{tabular}{|c|c|c|c|c|c|c|}
\hline Autor, Alto & LugzarEuterno & Objetivo & Instrumeuto & Metodologia & Idioma & Resultados \\
\hline Babakus y Mangold & $\begin{array}{l}\text { Estados Unidos } \\
\text { Se apbica en pscientes } \\
\text { dados de alta dentro de los } \\
\text { trece meses anteriores }\end{array}$ & $\begin{array}{l}\text { Vabiar la escala } \\
\text { SERVQUAL en un ambiente } \\
\text { bospitalario, adaptando el } \\
\text { cuestionario a las necesidades } \\
\text { de los servicios prestados en } \\
\text { un ertomo de salud. }\end{array}$ & SERVQUAL Motficsda & $\begin{array}{l}\text { Se crivis la encuesta } \\
\text { por cerreo postal a } \\
2036 \text { pacientes, bubo } \\
\text { una respuesta del } 22 \% \\
\text { y a medida que se } \\
\text { recilizis las encuestas } \\
\text { se depuraban las } \\
\text { preguitas adaptindolas } \\
\text { a las necesidades de } \\
\text { los pacientes. }\end{array}$ & Inglés & 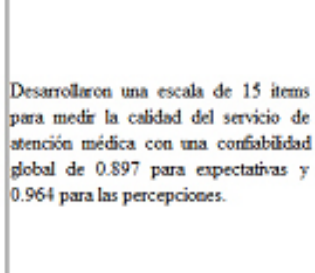 \\
\hline $\begin{array}{l}\text { Mira, Aranaz, } \\
\text { Rotriguez, et al. }\end{array}$ & $\begin{array}{l}\text { Espajis } \\
\text { Se aplica a los pacientes } 20 \\
\text { dias después de ser dados } \\
\text { de ath }\end{array}$ & $\begin{array}{l}\text { Vabidar el cuestionario } \\
\text { sERVQHO5 en la poblsción } \\
\text { esparlola. }\end{array}$ & SERVQHOS & $\begin{array}{l}\text { Se canvio la encuesta } \\
\text { por cerce postal a } \\
1424 \text { pacientes y se } \\
\text { obturo una respuesta } \\
\text { vilida de } 470 \text { pacientes } \\
\text { (tasa de respuesta de } \\
33 \% \text { ). }\end{array}$ & Español & 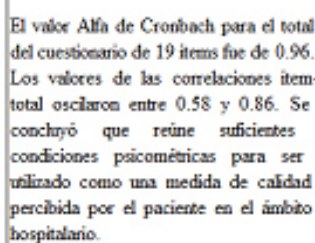 \\
\hline
\end{tabular}

TABLA 2 (CONT.)

Resumen de los resultados de algunos artículos relevantes sobre validación de instrumentos para evaluar la calidad percibida por los usuarios en los servicios de salud.

\begin{tabular}{|c|c|c|c|c|c|c|}
\hline Autor, Alio & LuzarEntorno & Objetivo & Instrumeuto & Metodologia & Idiom & Resultados \\
\hline $\begin{array}{l}\text { Cabrera, Londolo y } \\
\text { Beto }\end{array}$ & $\begin{array}{l}\text { Colombin } \\
\text { Usurios de servicios } \\
\text { efectivos o de urgescias }\end{array}$ & 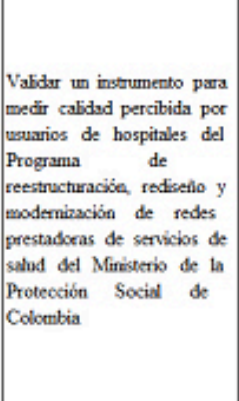 & PECASSUS & 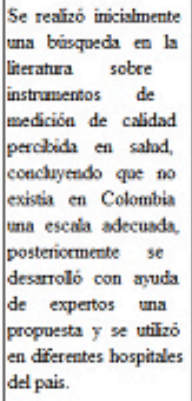 & Español & 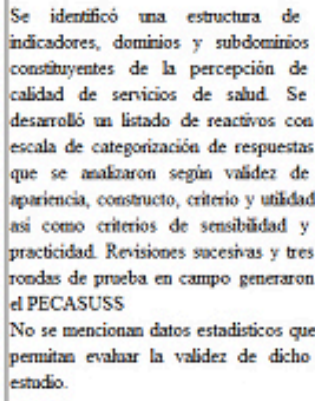 \\
\hline $\begin{array}{l}\text { Baragin } \quad y \\
\text { Manrigue }\end{array}$ & $\begin{array}{l}\text { Depurtamento de Boyacd, } \\
\text { Colombias } \\
\text { Se apbica en usuarios de } \\
\text { servicios quirurgicos }\end{array}$ & $\begin{array}{l}\text { Determinar la vabides del } \\
\text { cuestionario SERVQHOS } \\
\text { sobre catidad perctida en los } \\
\text { uesurios del servicio de } \\
\text { enfermeria }\end{array}$ & SERVQHOS & $\begin{array}{l}\text { Estudio metodolójico, } \\
\text { descriptivo y } \\
\text { transversal con andisis } \\
\text { multivariable de datos. } \\
\text { Se ranfizaron } 350 \\
\text { fonsularios. }\end{array}$ & Español & 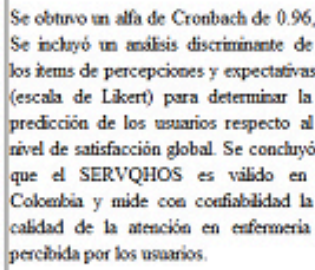 \\
\hline
\end{tabular}


TABLA 2 (CONT.)

Resumen de los resultados de algunos artículos relevantes sobre validación de instrumentos para evaluar la calidad percibida por los usuarios en los servicios de salud.

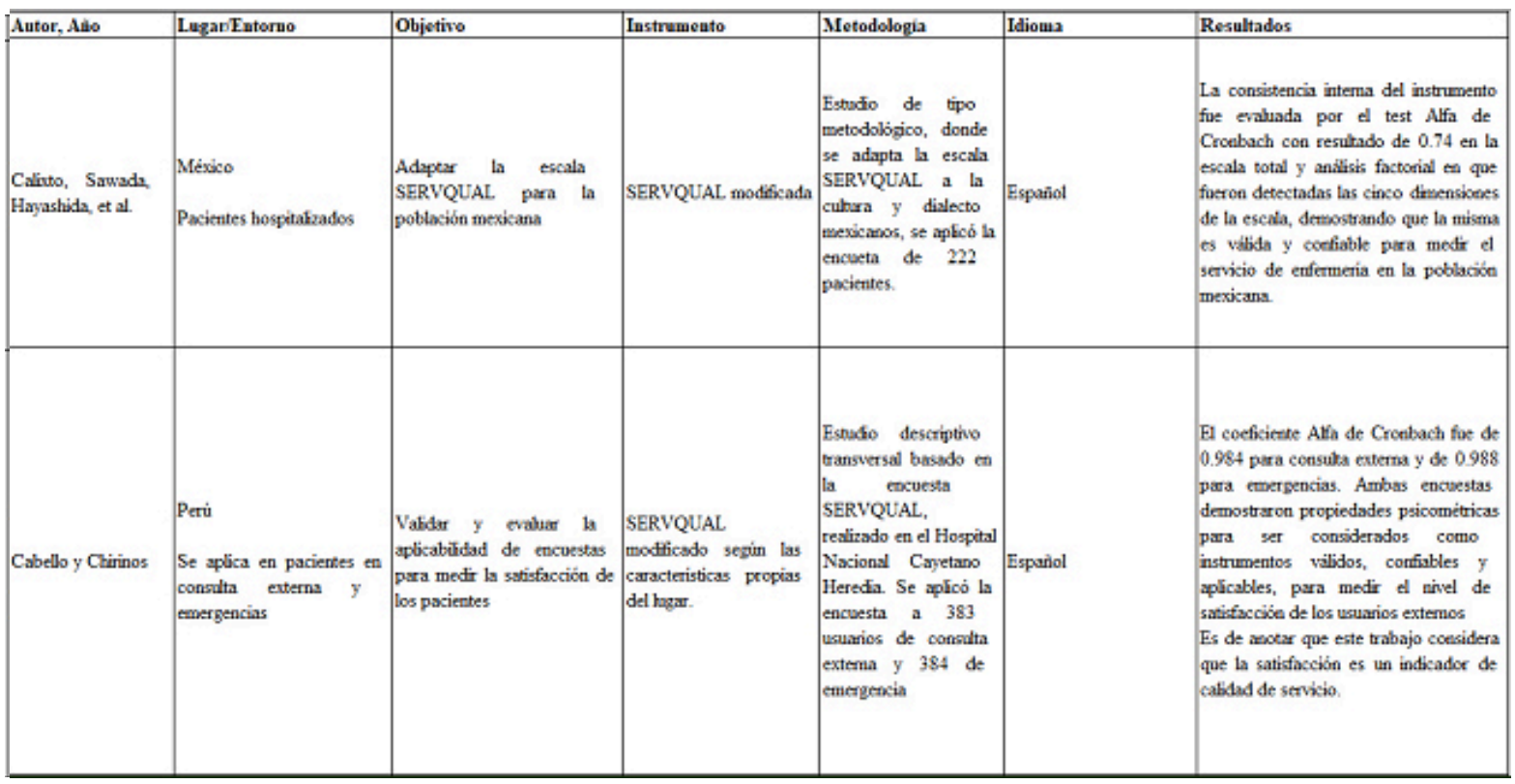

TABLA 2 (CONT.)

Resumen de los resultados de algunos artículos relevantes sobre validación de instrumentos para evaluar la calidad percibida por los usuarios en los servicios de salud.

\begin{tabular}{|c|c|c|c|c|c|c|}
\hline Autor, Alo & LugavEntorno & Objetivo & Instrumeuto & Metodologia & Idiom2 & Resultados \\
\hline $\begin{array}{l}\text { Rocha, Veiga, } \\
\text { Otrvera et al. }\end{array}$ & $\begin{array}{l}\text { Brasa } \\
\text { Pacientes hospitalzados en } \\
\text { el servicio de cirugia plistica }\end{array}$ & $\begin{array}{l}\text { Vabdar The Heath Service } \\
\text { Quality Scale midiendo el } \\
\text { coeficiente de correlación de } \\
\text { Pearson para medir la } \\
\text { asociación entra la versión en } \\
\text { portugués y la escala } \\
\text { SERVQUAL. }\end{array}$ & $\begin{array}{l}\text { The Health Service } \\
\text { Qualky Scale adspeada. }\end{array}$ & $\begin{array}{l}\text { Estudio transversal } \\
\text { observacional con } \\
\text { pscientes de tn } \\
\text { bospital püblico } \\
\text { universitario. Se tenia } \\
\text { una moestra de } 116 \\
\text { pacientes. }\end{array}$ & Portugués & 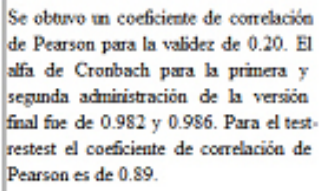 \\
\hline De La Hos Coerea & $\begin{array}{l}\text { Cudad de Modelin, } \\
\text { Colombita } \\
\text { No se ba reobado et } \\
\text { instrumento }\end{array}$ & 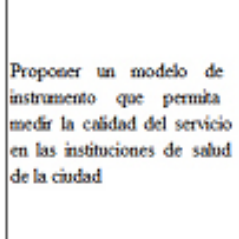 & SERVQUAL motificada & $\begin{array}{l}\text { Estudio de tipo } \\
\text { exploratorio, en ell que } \\
\text { a partir de la revisión } \\
\text { bubliografica se } \\
\text { joçone un modelo de } \\
\text { instrumento para medir } \\
\text { la calidad del servicio } \\
\text { en las instituciones de } \\
\text { salud de la ciadad }\end{array}$ & Espatiol & 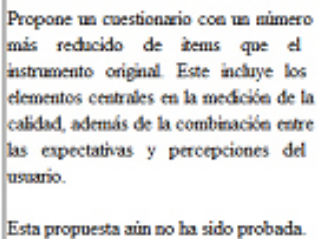 \\
\hline
\end{tabular}


TABLA 2 (CONT.)

Resumen de los resultados de algunos artículos relevantes sobre validación de instrumentos para evaluar la calidad percibida por los usuarios en los servicios de salud.

\begin{tabular}{|c|c|c|c|c|c|c|}
\hline Autor, Alio & LuzgabEutorno & Objotivo & Instrumento & Metodologia & Idiom & Resultados \\
\hline Büdogan e Iskendet & $\begin{array}{l}\text { Trrequia } \\
\text { Usuarios de tratamiernos } \\
\text { médicos en } 3 \text { centros de } \\
\text { sabud päblicos }\end{array}$ & 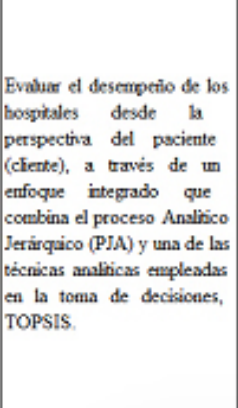 & $\begin{array}{l}\text { SERVQUAL, PJA, } \\
\text { TOPSIS }\end{array}$ & 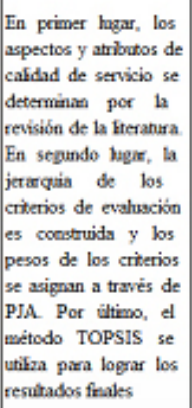 & Inglés & 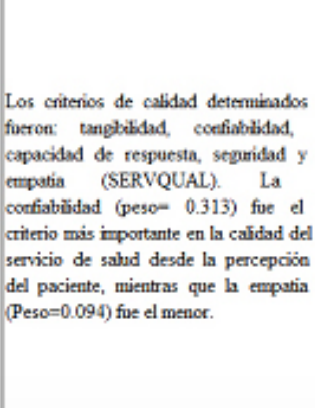 \\
\hline
\end{tabular}

\section{Discusión}

El primer estudio con el cual se validó el modelo Servqual en los servicios de salud fue el llamado Adapting the Servqual scale to Hospital Services: An Empirical Investigation, publicado en 1991. En este estudio se eliminaron algunos ítems del Servqual original que no eran adecuados para el entorno hospitalario, y que podían llevar a confusión de los pacientes. Esto, luego de un estudio de la escala original por parte de un panel de expertos. La escala modificada fue probada inicialmente en un grupo pequeño de pacientes. Este pretest indicó que algunas de las preguntas eran consideradas redundantes y que llevaban a una baja tasa de respuesta, por lo que la escala fue reducida a quince preguntas para los ámbitos de percepción y expectativas, respectivamente, evaluándose los cinco ámbitos de calidad expuestos anteriormente. Por último, se optó por la escala de cinco puntos de Likert, en vez de la siete como estaba formulado originalmente [13], y se obtuvo un resultado confiable en los cinco ámbitos para las expectativas y percepciones del paciente (tabla 3). 
TABLA 3

Confiabilidad para las subescalas de expectativas y percepciones Fuente: adaptación SERVQUAL, Emin Babacus y Glynn Mangold (13)

\begin{tabular}{|l|l|l|}
\hline \multirow{2}{*}{ Variable } & \multicolumn{2}{|c|}{ Coeficientes Alfa de Cronbach } \\
\cline { 2 - 3 } & \multicolumn{1}{|c|}{ Expectativas } & Percepciones \\
\hline Fiabilidad & 0.677 & 0.759 \\
\hline Capacidad de respuesta & 0.715 & 0.715 \\
\hline Seguridad & & 0.892 \\
\hline Empatia & 0.801 & 0.903 \\
\hline Elementos tangibles & 0.495 & 0.782 \\
\hline
\end{tabular}

También se analizó la confiabilidad de las combinaciones lineales de las cinco subescalas para evaluar la consistencia interna global de las mediciones de las expectativas y las percepciones. Los valores globales del coeficiente de alfa de Cronbach fueron 0.897 y 0.964 , lo que sugiere que hay unos niveles de consistencia interna adecuados. Dentro de la discusión del artículo se menciona que el instrumento Servqual posee una excelente confiabilidad de consistencia interna, con dos escalas de adecuada validez, una que habla de las expectativas de los pacientes del entorno hospitalario y otra de la percepción que tienen de su desempeño. También indican que la escala puede ser utilizada para evaluar la magnitud de la brecha entre expectativas y percepciones del paciente [13]. Finalmente, se concluye que Servqual es un instrumento estándar para medir la calidad del servicio funcional, es confiable y valido en el ambiente hospitalario (véase anexo 1).

Cada país tiene el número y los tipos de dimensiones útiles para evaluar la calidad de diferentes tipos de servicio de salud (públicos, privados, urgencias, urbanos, rurales etcétera), estas modificaciones también se basan en adaptaciones culturales y de lenguaje $[11,14,15,16,17,18,19]$. Una de las modificaciones más importantes de la escala Servqual es la escala Servqhos, realizada en España, y que mostró un mejor comportamiento que la escala Servqual en los servicios de salud. Esta propuesta reúne ventajas como un número más reducido de ítems (diecinueve) y, a diferencia del Servqual, cuenta con una escala de respuesta que combina expectativas y percepciones; por lo tanto, brinda mayor facilidad, rapidez y comprensión para los pacientes [20] (véase anexo 2).

Algunos autores también han intentado la incorporación al Servqual de métodos como el proceso analítico jerárquico (PJA) en servicios de salud, debido a que la percepción de la calidad del servicio se diferencia de forma individual en función de las preferencias de las personas, lo que trae consigo incertidumbre, subjetividad e imprecisión. Con esta propuesta integrada, además de tener los criterios de calidad, se construye una jerarquía de los criterios y se les asigna un peso a cada uno, lo que permite estimaciones más confiables de las expectativas de calidad [21,22]. Este es un modelo reciente que todavía exige futura investigación.

En Latinoamérica se han realizado adaptaciones del Servqual en países como México, Perú y Brasil que han demostrado ser instrumentos válidos para evaluar la calidad $(23,24,25,26)$. En nuestro país se ha intentado validar otras escalas. En el artículo "Validación de un instrumento para medir calidad percibida por usuarios de hospitales de Colombia” [3], se presenta un estudio que tenía como objetivo validar la escala 
Pecassus (percepción de calidad según usuarios de servicios de salud). Los autores realizaron inicialmente una búsqueda de la literatura y encontraron que en Colombia no se disponía de un instrumento validado para medir la percepción de la calidad en los servicios de salud en los pacientes. De acuerdo con los lineamientos propuestos en la literatura para desarrollar escalas de medición en salud, además de apoyarse en discusiones con profesores universitarios e investigadores acerca del tema, determinaron unos indicadores, dominios o factores inherentes a la percepción de los pacientes en los servicios de salud. Se generó una lista con ochenta aspectos referidos a calidad percibida, que después se aglutinaron en cuarenta preguntas, y posteriormente se procedió a validarlo en tres hospitales del área metropolitana de Medellín. Luego se realizó una prueba piloto en doce instituciones en Antioquia, Valle del Cauca, Bogotá y Barranquilla.

$\mathrm{Al}$ terminar este segundo paso, se adicionó una pregunta acerca de las instalaciones y se retiró una acerca del egreso de los pacientes. Finalmente, se realizó una última prueba en dos hospitales de Bogotá y se observaron excelentes resultados en cuanto al tiempo de aplicación, que no superaba los cinco minutos, la estabilidad de la forma de preguntar, la clara comprensión por el usuario de cada pregunta y las opciones de respuesta [3]. Posteriormente, los mismos autores, en un estudio publicado en el artículo "Calidad percibida por usuarios de hospitales del programa de reestructuración de redes de servicios de salud de Colombia”, establecieron la calidad percibida por usuarios con el instrumento Pecassus. Desafortunadamente, estos artículos no gozan de una rigurosidad estadística, y no permiten establecer la validez y confiabilidad de dichos resultados [27].

En Colombia también se ha validado la escala Servqhos, los resultados se presentaron en el artículo: "Validity and reliability of Servqhos for nursing Boyacá, Colombia", publicado en el año 2010. En este estudio se concluyó que el Servqhos es válido en Colombia y mide con confiabilidad la calidad de la atención en enfermería percibida por los usuarios [28].

Finalmente, en Colombia también se halló una propuesta de instrumento que incluyó un número más reducido de ítems que el instrumento original Servqual. Este instrumento reúne los elementos centrales en la medición de la calidad, además de la combinación entre las expectativas y las percepciones del usuario. Sin embargo, este instrumento aún no ha sido probado [29].

\section{Conclusión}

La medición en la calidad de servicios de salud es motivo de interés de muchas instituciones de salud a nivel mundial, ya que es parte fundamental de los servicios que se le brindan al paciente. Por esto, la participación del usuario resulta determinante para establecer procesos de mejoramiento y mediciones acerca de la calidad percibida en los servicios de salud. Durante la revisión bibliográfica realizada se encontraron estudios que validaron y aplicaron la escala Servqual y Servqhos en el ámbito hospitalario. Estos estudios mostraron una validez interna adecuada para dichas escalas, así como de las diferentes adaptaciones que se les realizó en los diferentes países en donde se aplicaron. Aunque en nuestro país se ha intentado validar escalas diferentes a las anteriormente mencionadas, no cuentan con un rigor estadístico importante que permita dar validez a los estudios señalados.

\section{Referencias}

1. Ross C, Frommelt G, Hazelwood L, Chang R. The role of expectations in patient satisfaction with medical care. J Health Care Mark. 1987; 7(4):16-26.

2. Tam J. Linking quality improvement with patient satisfaction: a study of a health service center. Mark Intell Plan. 2007;27(7):732-45.

3. Cabrera G, Londoño J, Bello L. Validación de un instrumento para medir calidad percibida por usuarios de hospitales de Colombia. Rev Salud Pública. 2008; 10(3):443-51. 
4. Losada M, Rodríguez A. Calidad del servicio de salud: una revisión a la literatura desde la perspectiva de marketing. Cuad Adm Bogotá. 2007; 20(34):237-58.

5. Parasuraman A, Zeithaml V, Berry L. A Conceptual model of service quality and its implications for future research. J Mark. 1985; (49):41-50.

6. Grönroos C. A service quality model and its marketing implications. Eur J Mark. 1984; 18(4):34-44.

7. Grönroos C. Service quality: the six criteria of good service quality. Rev Bus. 1988; (9):10-3.

8. Wilde Larsson B, Larsson G. Development of a short form of the Quality from the Patient's Perspective (QPP) questionnaire. J Clin Nurs. 2002; 11(5):681-7.

9. Velandia F, Ardón N, Jara MI. Satisfacción y calidad: análisis de la equivalencia o no de los términos. Rev Gerenc y Políticas Salud. 2007; 6(13):139-68.

10. Beattie M, Murphy D, Atherton I, Lauder W. Instruments to measure patient experience of healthcare quality in hospitals: a systematic review. Syst Rev. 2015; 4(97):1-21.

11. Mohammed K, Rajjo T, Prathibha V. Creating a patient-centered health care delivery system: A systematic review of health care quality from the patient perspective. Am J Med Qual. 2016; 31(1):12-21.

12. Parasuraman A, Zeithaml V, Berry L. Servqual: A multiple-item scale for measuring consumer perceptions of service quality. J Retail. 1988; 64(1):12-40.

13. Babakus E, Mangold W. Adapting the Servqual scale to hospital services: an empirical investigation. Heal Serv Res. 1992; 26(6):767-86.

14. Akhade G, Jaju S, Lakhe R. Critical review of global practices in measuring healthcare service quality. Int J Eng Res Technol. 2016; 5(2):762-9.

15. Qin H, Prybutok V. A quantitative model for patient behavioral decisions in the urgent care industry. Socioecon Plann Sci. 2013; (47):50-64.

16. Qin H, Prybutok G, Prybutok V, Wang B. Quantitative comparisons of urgent care service providers. Int J Health Care Qual Assur. 2015; 28(6):574-94.

17. Min L, Douglas B, Cheng-Yu H, Xiang-Chan L, Ying-Chu Z, Xing-Hua W, et al. Evaluating patients'perception of service quality at hospitals in nine Chinese cities by use of the ServQual scale. Asian Pac J Trop Biomed. 2015; 5(6):497-504.

18. Purcărea V, Raluca I, Mădălina C. The assessment of perceived service quality of public health care services in Romania using the Servqual Scale. Procedia Econ Financ. 2013; (6):573-85.

19. Ganesh N, Jaju S, Lakhe R. Identification of service quality attributes for healthcare services. Int J Eng Trends Technol. 2016; 32(6):37-44.

20. Mira J, Aranaz J, Rodríguez J, Buil J, Castell M, Vitaller J. Servqhos: un cuestionario para evaluar la calidad percibida de la atención hospitalaria. Med Prev. 1998; (4):12-8.

21. Birdogan B, İskender P. An integrated evaluation model for service quality of hospitals: a case study from Turkey. J Mult-Valued Log Soft Comput. 2015; (24):453-74.

22. Lupo T. A fuzzy framework to evaluate service quality in the healthcare industry: An empirical case of public hospital service evaluation in Sicily. Appl Soft Comput. 2016; (40):468-78.

23. Calixto M, Sawada N, Hayashida M, Mendes I, Trevizan M, Godoy S. Escala Servqual: validación en población mexicana. Enferm. 2011; 20(3):526-33.

24. Pedraza N, Bernal I, Lavín J, Lavín J. La calidad del servicio: Caso UMF. Concienc Tecnológica. 2015; (49):39-45.

25. Cabello E, Chirinos J. Validation and applicability of Servqual modified survey to measure external user satisfaction in health services. Rev Med Hered. 2012; 23(2):88-95.

26. Rocha L, Veiga D, e Oliveira P, Song E, Ferreira L. Health service quality scale: Brazilian Portuguese translation, reliability and validity. BMC Heal Serv Res. 2013; 13(24):1-5.

27. Gustavo C, León B, Jaime L. Calidad percibida por usuarios de hospitales del Programa de Reestructuración de Redes de Servicios de Salud de Colombia. Rev Salud Pública. 2008; 10(4):593-604. 
28. Barragán J, Manrique F. Validity and reliability of Servqhos fornursing Boyacá, Colombia. Av Enferm. 2010; 28(2):48-61.

29. De La Hoz Correa P. Propuesta de aplicación de la escala Servqual en el sector salud de Medellín. CES Salud Pública. 2014; (5):107-16.

\title{
Anexo 1: The Servqual Instrument
}

\author{
Babakus E, Mangold W [13]
}

Expectations Scale (a five-point Likert response format)

Tangibles

El. Hospitals should have up-to-date equipment.

E2. Hospitals' physical facilities should be visually appealing.

E3. Hospital employees should appear neat.

Reliability

E4. Hospitals should provide their services at the time they promise to do so.

E5. When patients have problems, hospital employees should be sympathetic and reassuring.

E6. Hospitals should be accurate in their billing.

Responsiveness

E7. Hospital employees should tell patients exactly when services will be performed.

E8. It is realistic for patients to expect prompt service from hospital employees.

E9. Hospital employees should always be willing to help patients.

Assurance

E10. Patients should be able to feel safe in their interactions with hospital employees.

E11. Hospital employees should be knowledgeable.

E12. Hospital employees should be polite.

E13. Hospital employees should get adequate support from their employers to do their jobs well.

Empathy

E14. Hospital employees should be expected to give patients personal attention.

E15. It is realistic to expect hospitals to have their patients' best interests at heart.

Perceptions Scale (a five-point Likert response format)

Tangibles

P1. XYZ has up-do-date equipment.

P2. XYZ's physical facilities are visually appealing.

P3. XYZ's employees appear neat.

Reliability

P4. XYZ provides its services at the time it promises to do so.

P5. When patients have problems, XYZ's employees are sympathetic and reassuring.

P6. XYZ is accurate in its billing.

Responsiveness

P7. XYZ employees tell patients exactly when services will be performed.

P8. Patients receive prompt service from XYZ's employees.

P9. XYZ's employees are always willing to help patients

Assurance

P10. Patients feel safe in their interactions with XYZ's employees.

P11. XYZ's employees are knowledgeable.

P12. XYZ's employees are polite. 
P13. Employees get adequate support from XYZ to do their jobs well.

\section{Empathy}

P14. XYZ's employees give patients personal attention.

P15. XYZ has patients' best interests at heart.

"In terms of the quality of care received, what is your overall impression of hospital?" (The overall quality perceptions statement was measured on a five-point scale with end points labeled "very good" and "very poor.")

"If I were to find myself in the same situation I was in when I went to hospital, I would want to receive my treatment there again." (The intention-to-return statement, measured on a five-point "strongly agree"-"strongly disagree" scale,)

\section{Anexo 2: Servqhos}

Mira J, Aranaz J, Rodríguez J, et al. [20]

Servqhos:

Encuesta de opinión sobre la calidad de la atención hospitalaria

Estimado Sr./Sra.: Quienes trabajamos en el Hospital __ estamos interesados en conocer su opinión acerca de la calidad de servicio hospitalario que se ha ofrecido durante su estancia en el mismo. Por este motivo nos permitimos dirigirnos a Vd. con el propósito de solicitar su colaboración, con la seguridad de que su opinión y ayuda será de gran utilidad para que podamos hacerlo cada vez mejor.

Por supuesto que su colaboración es totalmente voluntaria y completamente anónima. Todo lo que diga será tratado de forma absolutamente confidencial.

Una vez que haya contestado a todas las preguntas puede introducir la encuesta en el sobre que le hemos facilitado y enviarla por correo. El sobre lleva la dirección y franquicia para su comodidad. Nos permitimos sugerirle que responda cuanto antes para que no se olvide de hacerlo.

Le agradecemos muy sinceramente su interés al leer esta carta y su atención para con nuestro trabajo, con la seguridad de que será en beneficio de todos.

\section{EL DIRECTOR DEL HOSPITAL}

Basándose en su experiencia señale si la calidad de la asistencia sanitaria que le ha proporcionado el Hospital ha sido mejor o peor de lo que Ud. esperaba. Si cree, por ejemplo, que ha sido MUCHO PEOR de lo que esperaba ponga una X en la casilla 1 . Si ha sido MUCHO MEJOR de lo que esperaba ponga la $\mathrm{x}$ en la casilla 5 , y así sucesivamente 
TABLA 4

La calidad de la asistencia sanitaria ha sido...

\begin{tabular}{|c|c|c|c|c|c|}
\hline \multicolumn{6}{|c|}{ La calidad de la asistencia sanitaria ha sido } \\
\hline & $\begin{array}{l}\text { Mucho peor } \\
\text { de lo que } \\
\text { esperaba }\end{array}$ & $\begin{array}{l}\text { Peor de lo que } \\
\text { esperaba }\end{array}$ & $\begin{array}{c}\text { Como me lo } \\
\text { esperaba }\end{array}$ & $\begin{array}{l}\text { Mejor de lo } \\
\text { que esperaba }\end{array}$ & $\begin{array}{c}\text { Mucho mejor } \\
\text { de lo que } \\
\text { esperaba }\end{array}$ \\
\hline Clave respuesta & 1 & 2 & 3 & 4 & 5 \\
\hline \multicolumn{6}{|c|}{ En el Hospital } \\
\hline $\begin{array}{l}\text { La tecnología de los equipos médicos para } \\
\text { los diagnósticos y tratamientos ha sido }\end{array}$ & 1 & 2 & 3 & 4 & 5 \\
\hline $\begin{array}{l}\text { La apariencia (limpieza y uniforme) del } \\
\text { personal ha sido }\end{array}$ & 1 & 2 & 3 & 4 & 5 \\
\hline $\begin{array}{l}\text { Las indicaciones (señalizaciones) para } \\
\text { orientarse y saber a dónde ir en el hospital } \\
\text { han sido }\end{array}$ & 1 & 2 & 3 & 4 & 5 \\
\hline $\begin{array}{l}\text { El interés del personal por cumplir lo que } \\
\text { promete ha sido }\end{array}$ & 1 & 2 & 3 & 4 & 5 \\
\hline $\begin{array}{l}\text { El estado en que están las habitaciones del } \\
\text { hospital (apariencia, comodidad) ha sido }\end{array}$ & 1 & 2 & 3 & 4 & 5 \\
\hline $\begin{array}{l}\text { La información que los médicos } \\
\text { proporcionan ha sido }\end{array}$ & 1 & 2 & 3 & 4 & 5 \\
\hline $\begin{array}{l}\text { El tiempo de espera para ser atendido por } \\
\text { un médico ha sido }\end{array}$ & 1 & 2 & 3 & 4 & 5 \\
\hline La facilidad para llegar al hospital ha sido & 1 & 2 & 3 & 4 & 5 \\
\hline $\begin{array}{l}\text { El interés del personal por solucionar los } \\
\text { problemas de los pacientes ha sido }\end{array}$ & 1 & 2 & 3 & 4 & 5 \\
\hline $\begin{array}{l}\text { La puntualidad de las consultas médicas } \\
\text { ha sido }\end{array}$ & 1 & 2 & 3 & 4 & 5 \\
\hline $\begin{array}{l}\text { La rapidez con que consigues lo que se } \\
\text { necesita o se pide ha sido }\end{array}$ & 1 & 2 & 3 & 4 & 5 \\
\hline $\begin{array}{l}\text { La disposición del personal para ayudarle } \\
\text { cuando lo necesita ha sido }\end{array}$ & 1 & 2 & 3 & 4 & 5 \\
\hline $\begin{array}{l}\text { La confianza (seguridad) que el personal } \\
\text { transmite a los pacientes ha sido }\end{array}$ & 1 & 2 & 3 & 4 & 5 \\
\hline \begin{tabular}{|l|} 
La amabilidad (cortesia) del personal en su \\
trato con la gente ha sido
\end{tabular} & 1 & 2 & 3 & 4 & 5 \\
\hline $\begin{array}{l}\text { La preparación del personal(capacitación) } \\
\text { para realizar su trabajo ha sido }\end{array}$ & 1 & 2 & 3 & 4 & 5 \\
\hline $\begin{array}{l}\text { El trato personalizado que se le da a los } \\
\text { pacientes ha sido }\end{array}$ & 1 & 2 & 3 & 4 & 5 \\
\hline $\begin{array}{l}\text { La capacidad del personal para } \\
\text { comprender las necesidades de los } \\
\text { pacientes ha sido }\end{array}$ & 1 & 2 & 3 & 4 & 5 \\
\hline $\begin{array}{l}\text { La información que los médicos dan a los } \\
\text { familiares ha sido }\end{array}$ & 1 & 2 & 3 & 4 & 5 \\
\hline $\begin{array}{l}\text { El interés del personal de enfermeria por } \\
\text { los pacientes ha sido }\end{array}$ & 1 & 2 & 3 & 4 & 5 \\
\hline
\end{tabular}

Marque con una X la casilla que mejor refleje su opinión 
TABLA 5

Satisfacción global

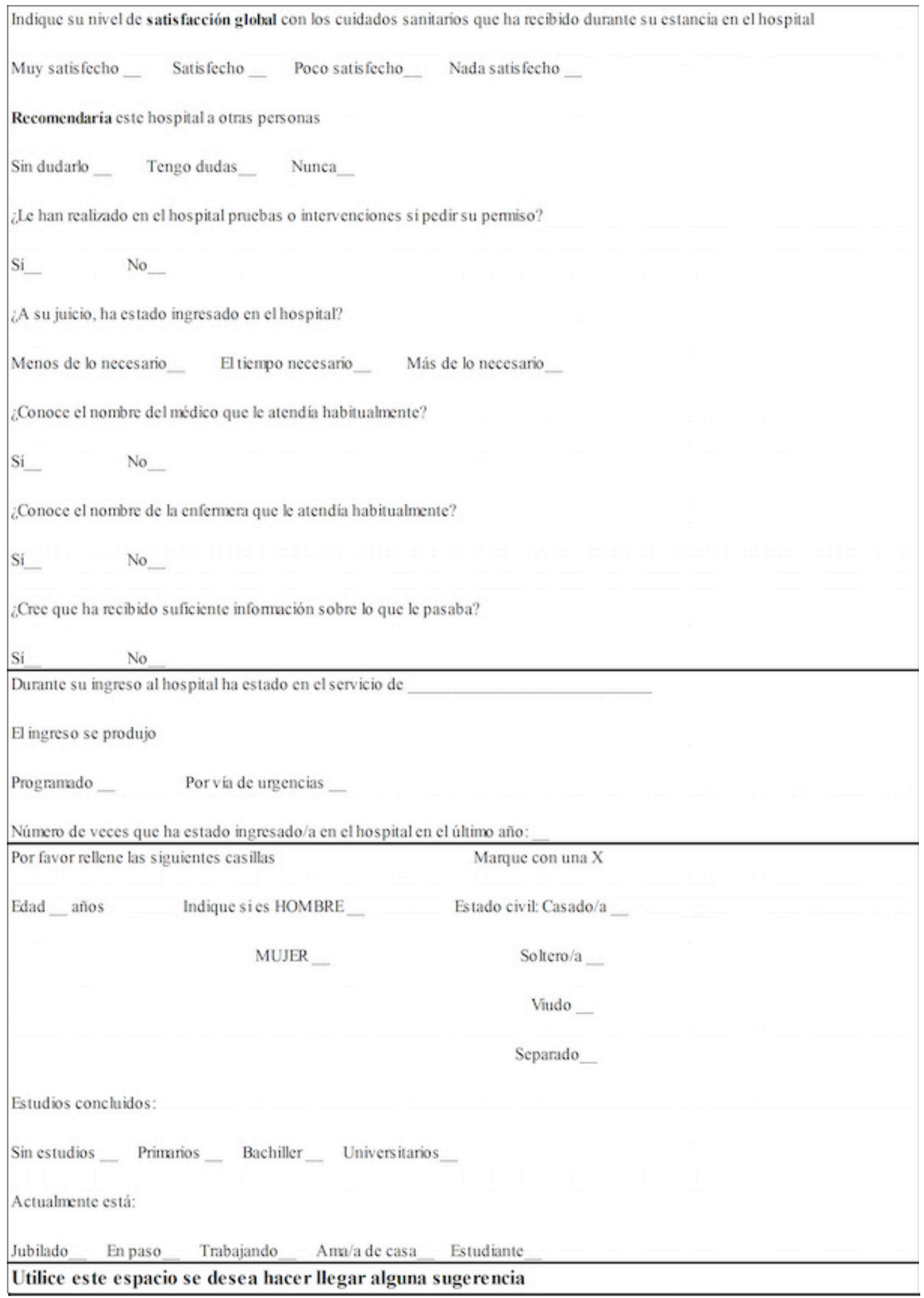




\section{Notas}

* Artículo de revisión

Licencia Creative Commons CC BY 4.0

Como citar este artículo: Henao Nieto DE, Giraldo Villa A, Yepes Delgado CE. Instrumentos para evaluar la calidad percibida por los usuarios en los servicios de salud. Rev Gerenc Polít Salud. 2017; 17(34): 1-21. https://doi.org/10.11144/Javeriana.rgps 17-34.iecp 